\title{
Insider Threats in Emerging Mobility-as-a-Service Scenarios
}

\author{
Franco Callegati \\ University of Bologna \\ franco.callegati@unibo.it \\ Andrea Melis \\ University of Bologna \\ a.melis@unibo.it
}

\author{
Saverio Giallorenzo \\ University of Bologna, INRIA \\ saverio.giallorenzo2@unibo.it \\ Marco Prandini \\ University of Bologna \\ marco.prandini@unibo.it
}

\begin{abstract}
Mobility as a Service (MaaS) applies the everything-asa-service paradigm of Cloud Computing to transportation: a MaaS provider offers to its users the dynamic composition of solutions of different travel agencies into a single, consistent interface.

Traditionally, transits and data on mobility belong to a scattered plethora of operators. Thus, we argue that the economic model of MaaS is that of federations of providers, each trading its resources to coordinate multi-modal solutions for mobility. Such flexibility comes with many security and privacy concerns, of which insider threat is one of the most prominent. In this paper, we follow a tiered structure - from individual operators to markets of federated MaaS providers - to classify the potential threats of each tier and propose the appropriate countermeasures, in an effort to mitigate the problems.
\end{abstract}

\section{INTRODUCTION}

The term Cloud Computing denotes a dynamic infrastructure where users access services without regard to where the services are hosted [1]. The concept of Mobility as a Service (MaaS) [2] takes inspiration from such a model and brings it into the context of transportation. In Cloud Computing, the architecture that runs the services is dynamic and transparent to users. Likewise, MaaS hides a dynamic composition of solutions provided by different travel agencies behind a consistent interface. Hence, MaaS users experience traveling as provided by a single agency.

Due to regulatory and logistic issues, mobility resources are administrated and owned by a scattered plethora of mobility operators (traditional travel agencies and providers of data for mobility). Thus, we argue that the leading economic model of MaaS markets is that of federations of mobility operators, each trading its resources. In such a federated market, operators can dynamically partner with each other, still preserving their individual autonomy and without the need for a centralized regulation authority. On these premises, we are currently developing a ServiceOriented platform, called Smart Mobility for All ${ }^{1}$ (SMAII), built on the concept of federated Cloud Computing [3], [4] and purposed to support liquid markets for transportation.

1. https://github.com/small-dev/SMAll.Wiki/wiki
During the development of SMAll and through the collaboration with our industrial partners (public administrations, local travel agencies, etc.), we identified and analyzed many security issues spanning from a single operator to a federation of operators. In this context, we deem malicious insider activity one of the most prominent, spanning from standard threats against cloud installations [5] to insider issues specific to the contexts of mobility and of markets of services.

Motivation. Fig. 1 depicts a cross section of an instantiation of SMAll, where the colored entities outside of the boundaries of SMAll (bordered with double lines) are public transportation agencies, private companies, on-line communities, and MaaS operators.

Even when considered in isolation, the agents in the platform already entail well-known threats due to insider activity. For example, the City Bus Operator represents a threat to the privacy of drivers since GPS positioning can reveal sensitive information on their conduct, which is forbidden under some legislation; however, also drivers represent an insider threat to the Bus Operator: they can disable the GPS device on their vehicles, compromising the reliability of the GPS positioning system and that of the other services that depend on $i^{2}$ (e.g., the Bus Delays service that estimates bus arrivals based on vehicle GPS positions). Finally employees can manipulate the services and their data, damaging the company by extracting restricted information or causing outages.

Broadening our scope to federated interactions, we focus on the MaaS Operator in Fig. 1 that, for example, deploys a Journey Planner service for providing dynamic multi-modal trips to users. The service orchestrates other federated services in SMAll: it uses information on scheduling, availability, disruptions, and the position of buses, trains, and on-demand cars.

As expected, the threats highlighted for single operators surface (and possibly combine) to higher-level

2. The issues are far from being just speculative, as we actually encountered them collaborating with one of our industrial partners. 


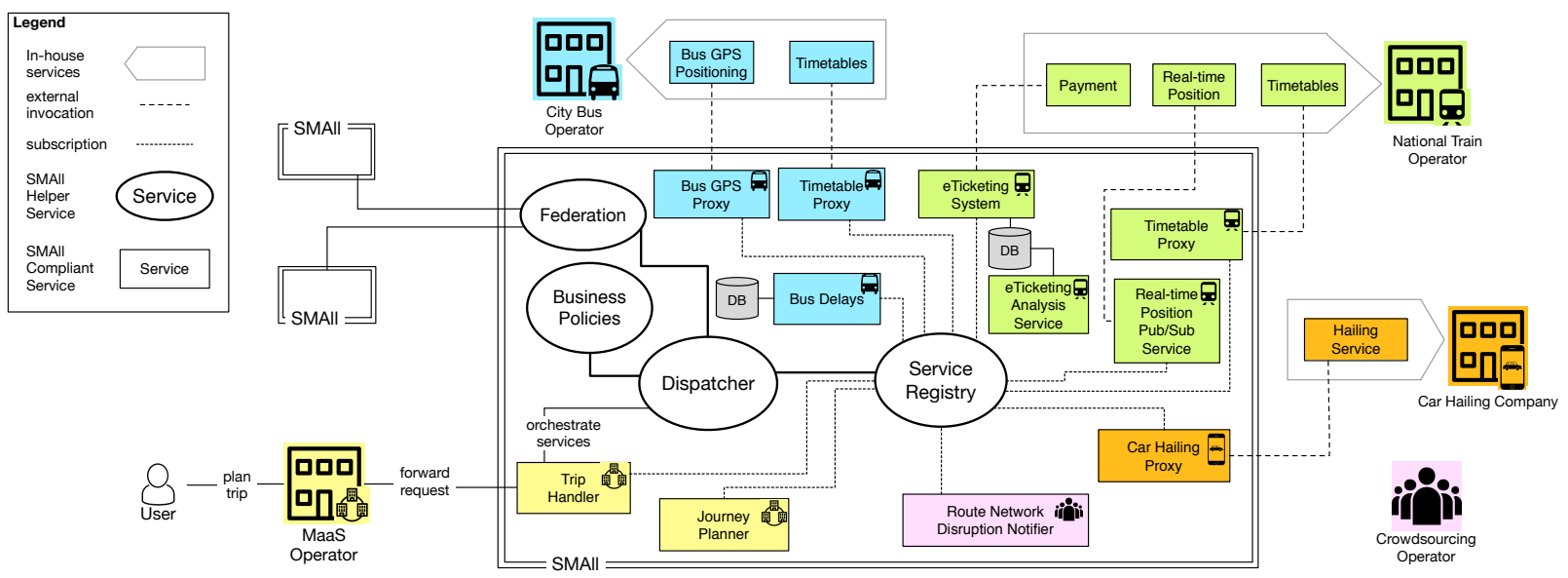

Figure 1. Example of the SMAll architecture.

federated scenarios. Consider the case in which the City Bus Operator allows the MaaS Operator to access the Bus GPS Proxy service. With the raw data on the real-time position of buses, the MaaS Operator can undertake many malicious activities to the detriment of the Bus Operator, e.g., passing relevant information to competitors of the Bus Operator. Another important threat comes from the extraction of sensitive information from aggregated/anonymized data. Aware of the threat posed by the Bus GPS Proxy service, the Bus Operator markets only its Bus Delays service. However, also aggregated data like the temporal approximation of the arrival of buses might let the MaaS Operator extract [6] the actual position of vehicles (possibly optimizing the accuracy of the extraction [7]).

Contribution. As exemplified, in the context of MaaS operators, the definition of what an insider is can assume subtle nuances depending on the considered scenario. In this work, guided by our experience with the development of SMAll, we describe the security issues concerning insiders within such a federated market of services. We develop our treatment following a tiered view of MaaS markets, called the MaaS Stack [8], summarized in $\S 2$. In $\S 3$, we consider each tier of the MaaS Stack, we define what an insider is for each of them, we analyze the related threats, and we describe the possible countermeasures.

\section{The MaAS Stack: An OVerview}

In this section we briefly overview the MaaS Stack (Fig. 2), a structured view that we assembled to guide the development of SMAll. In $\S 3$ we use the MaaS Stack to analyze the insider threats of each tier.

Tier I I eMobility Operators. The first tier of the MaaS Stack is that of eMobility Operators. An eMobility Operator is an entity that owns, administrates, and exposes software functionalities regarding mobility, provided in a machine-readable form. In tier I of the MaaS Stack, eMobility Operators are considered in isolation (i.e., not using and integrating the services of other operators).
For example, the National Train Operator represented in Fig. 1 is an eMobility Operator that owns services for purchasing tickets, accessing timetables, and receiving real-time position of vehicles.

Tier II I Business Intelligence. The second tier of the MaaS Stack still focuses on single eMobility operators but it enriches the taxonomy of services with the category of Business Intelligence. These services are not meant for users but for eMobility operators; they span over first-tier services by monitoring and analyzing their usages. Business Intelligence services provide insight on the performances of eMobility operators. For example, the eTicketing Analysis Service (Fig.1) of the Train Operator can suggest to the latter new pricing policies as well as reporting rarely used routes that could be merged/discarded.

Tier III I MaaS Operators. The last tier of the MaaS Stack is that of MaaS Operators, i.e., eMobility operators that federate and integrate their services with those of other eMobility operators. Each MaaS operator provides to its users information and transit services of other operators as its own. The principle resembles that of "roaming" in GSM phone networks [9], where users connect through the services of another phone company when traveling outside the geographical coverage area of the home network. The MaaS Operator represented in Fig. 1 can federate with the National Train Operator and the City Bus Operator and it can offer multi-modal journeys that span different means of transportation and have nation- to city-wide scopes. This example introduces the last fundamental element of the third tier of the MaaS Stack: Clearing services to account for federated usages and compensate operators according to the established Business Policies.

To support the mentioned features in SMAll, we are currently developing and integrating components to deploy services, to support the definition and enforcement of business and clearing policies, and to federate many instances of the platform. During the development, we recognized and investigated security issues derived from the openness 


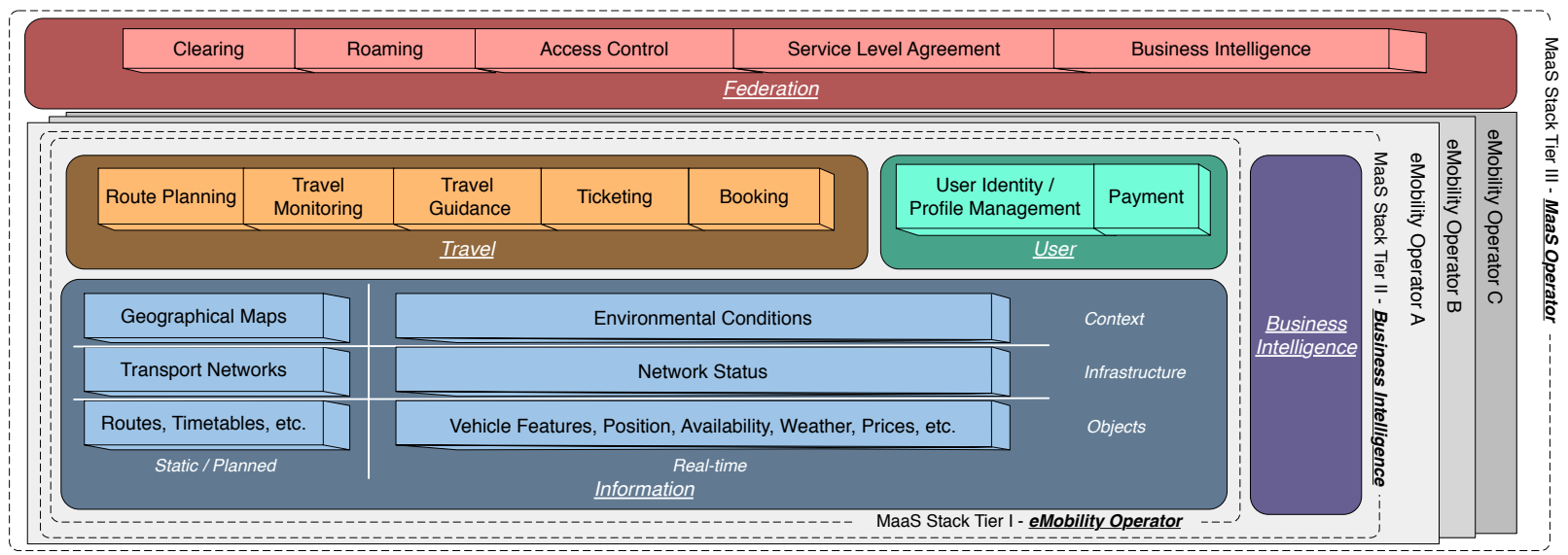

Figure 2. The MaaS Stack

of our federated platform. In the next section, we consider each tier of the MaaS Stack, we define what an insider is for each of them, we analyze the related threats, and we describe the possible amendments to counteract them.

\section{INSIDER THREATS IN MAAS SCENARIOS}

Statistically, insider threats are one of the most expensive security issues for business companies [10]. One prominent reason of these expensive outcomes is that companies did not foresee all possible malicious insider activities [11]. Indeed, the problem is not the lack of proper countermeasures as much as the difficulty of identifying a malicious insider in the first place. Literature abounds with guidelines and principles aimed at providing general descriptions of the context and the identity of the insiders [12], [13]. However, experts agree that the strong contextual variance of threats [14] makes providing a general yet precise identification of all possible insiders difficult.

Thus, we deem useful to share the experience we gained in the context of services for mobility (both at software and physical level). Moreover, our background on the development of SMAll provides insights on the possible threats deriving from federated cloud architectures, built for deploying, publishing, and trading services. Federated clouds have been already analyzed in literature [5], [15], however we deem important to include the related threats in the frame of the emerging Mobility-as-a-Service scenario.

In $\S 3.1-3.3$, we illustrate, for each tier of the MaaS Stack (cf. Fig. 2), the insiders, the related attacks, and the possible countermeasures, as found in the state of the art and implemented in SMAll. In Fig. 3 we report a table that summarizes our findings. Agents and threats are classified according to the categories identified by Casey in [16] and the CERT technical report [17].

We dedicate the last paragraph of this Section to a brief description of the methodology we followed to recognize the threats and the respective countermeasures.
Methodology. As mentioned, adopting a precise definition of insider may hinder the identification of threats specific to particular contexts. Therefore, in our investigation, we prefer to look at insiders from a general point of view [18]:

A trusted entity that is given the power to violate one or more rules in a given security policy [...] the insider threat occurs when a trusted entity abuses that power.

This definition hints that an insider is determined by the role played as member of a system and related to the deployed control rules and the pursuable malicious goal(s). In our context, the most classic scenario is one where the insider is within the service of the victim, e.g., a programmer that manipulates the behavior and the data of a service. However, orchestrations spanning many providers, hallmark of the SMAll platform, lead to subtle yet relevant threats. Consider the case of federated partners. On one hand, the provider of a service exposes itself to threats posed by members that use its service security issues span from misuse of information extracted from the service to over-usages that entail unforeseen costs or outages - on the other hand, an agent that orchestrates services of other partners is a man-in-the-middle able to leak private information, counterfeit data or use its vantage point to extract strategic patterns from partners.

Regarding countermeasures, we structured our analysis of the possible alternatives following the review compiled by Hunker and Probst [19], encompassing the three approaches: i) Prevention, comprising the definition of strong access control rules, data management systems (including data masking and data camouflage), and mechanisms to guarantee data provenance and data trustworthiness; ii) Detection, that usually goes hand-in-hand with dissuasion mechanisms such as techniques of data management and service invocation that make abuses extremely expensive in terms of computing power; iii) Mitigation, that exploits auditing and monitoring techniques, based on machine learning, to automatically identify and react to insiders. 


\begin{tabular}{|c|c|c|c|c|}
\hline Tier & Agent & \begin{tabular}{|c|} 
Agent Type \\
\end{tabular} & \begin{tabular}{|l} 
Insider Threat \\
\end{tabular} & Event Type \\
\hline \multirow{7}{*}{$1 \& 2$} & \multirow{2}{*}{ User } & \multirow{2}{*}{$\begin{array}{l}\text { Competitor, Untrained/Distracted Insider, } \\
\text { Outward Sympathizer }\end{array}$} & Fake Data Injection & Sabotage \\
\hline & & & Service Behavior Manipulation & Product Alteration, Sabotage \\
\hline & Unauthorized Guests & Competitor, Theft, Activist & Man-in-the-middle Attack & Misuse \\
\hline & Developers & Competitor, Partner, Disgruntled Insider & User Impersonation & Sabotage, Espionage, Misuse \\
\hline & Service Administrators & $\begin{array}{l}\text { Partner, Disgruntled Insider, } \\
\text { Untrained/Distracted Insider, Supplier }\end{array}$ & Insider Impersonation & Sabotage, Espionage, Misuse \\
\hline & Service Managers & $\begin{array}{l}\text { Partner, Disgruntled Insider, } \\
\text { Untrained/Distracted Insider, Supplier }\end{array}$ & Crowdsourcing Attacks & Sabotage, Financial Fraud \\
\hline & $\begin{array}{l}\text { Agent after privilege } \\
\text { escalation }\end{array}$ & Activist, Competitor & $\begin{array}{l}\text { Data Leakage } \\
\text { - Accidental } \\
\text { - Data Theft } \\
\text { - Resale of Data and Access } \\
\text { - Business Intelligence Data Theft }\end{array}$ & $\begin{array}{l}\text { IP Theft, Opportunistic Data Theft, Physical Theft, } \\
\text { Accidental Leak }\end{array}$ \\
\hline \multirow{7}{*}{3} & \multirow{5}{*}{ Federated MaaS Member } & \multirow{5}{*}{ Competitors Nation State Partner Supplier } & $\begin{array}{l}\text { Data Manipulation, Trustability, Tampering of } \\
\text { Data Provenance, Data Trustworthiness }\end{array}$ & Financial Fraud, Product Alteration \\
\hline & & & Service Behavior Manipulation & Financial Fraud, Product Alteration \\
\hline & & & Composition of Unverified Services and Data & Misuse, Sabotage, Espionage, Product Alteration \\
\hline & & & Denial of Service & Sabotage \\
\hline & & & Service Workflow Manipulation & Misuse, Sabotage, Espionage, Product Alteration \\
\hline & MaaS Competitor & Nation State, Partner, Supplier & \multirow{2}{*}{$\begin{array}{l}\text { Data Analysis: } \\
\text { - Pattern Extraction } \\
\text { - Data Mining } \\
\text { - Data Exploitation trough data crossing }\end{array}$} & \multirow[b]{2}{*}{$\begin{array}{l}\text { Accidental Leak, Opportunistic Data Theft Espionage, } \\
\text { Financial Fraud }\end{array}$} \\
\hline & Helper Service & $\begin{array}{l}\text { Competitors, Nation State, Partner, } \\
\text { Supplier }\end{array}$ & & \\
\hline
\end{tabular}

Figure 3. Summary table comparing the tiers of the MaaS Stack to the related insider threats.

\section{MaaS Stack | Tier I}

As reported in $\S 2$, the first tier of the MaaS Stack focuses on single eMobility operators and categorizes their services. In this tier, the ecosystem of services has a flat structure and all members play the same role of providers, without any interaction between each other. Here, insiders can be pinpointed within two types: i) users authorized to interact with services and ii) the managers (also seen as owners) of the services. In the reminder, we call Users the members of the first type and Managers the members of the second one. The distinction between the two types is trivial: while Users have limited access to data and functionalities of a service, Managers can have full or partial control (depending on the responsibility level) over the life-cycle of the service and its resources.

Users allowed to interact with SMAll services can basically pose two types of threats: $i$ ) perform fake data injection (for crowdsourcing-based services) and ii) sharing the access to the services or to the respective data. Users can also exploit vulnerabilities to acquire Manager privileges (configuring an Insider Impersonation threat). However, we do not include a discussion on these kind of attacks as they coincide with those described for Managers. Regarding Managers, their main threats comprise:

- manipulation of the behavior of a service, i.e., the computations done by a service;

- manipulation of the workflow among services, i.e., the flow of information among services;

- stealing data, metadata, and performing malicious analyzes;

- exposing sensitive information.

Following the first tier of the MaaS Stack, we describe the possible insider attacks of each category of services.

\section{Information}

The category of Information spans from basic services that publish raw data (e.g., timetables or the position of vehicles) to higher-level services that elaborate raw data to extract new information (e.g., the expected delay of buses whose calculation requires the position of a vehicle and its scheduled plan). Notably, since Information services orchestrate other services to calculate and publish these refined data, they are subject to Service Workflow Manipulation and Composition of Unverified Services and Data threats. We omit to present these issues in this Section and refer the discussion to $§ 3.1 .2$.

Data Leakage. Data leakage is the accidental distribution of private or sensitive data to unauthorized entities [20]. In SMAll, both Users and Managers can cause data leakage. Users can share data to other, non-authorized Users. Similarly, Users can also share their access to services, which could lead to data leakage but also to other type of threats like User Impersonation. As expected, data leakage becomes even more serious when considered for Managers that can share or steal sources unreachable by users.

Countermeasures. Data leakage poses a serious issue in open networks where the transition of data is not regulated nor monitored in their path. In these regards, SMAll holds a privileged position. In fact, all communications among the services in the platform happen through the Dispatcher (cf. Fig 1), which can $\log$ the quality and quantity of information required by all Users. Obviously, this guarantee ceases when data exits the platform. The same tracing system applies also to Managers.

Crowdsourcing Attacks. Users can perform insider attacks on crowdsourcing services. These services handle data 
streamed from sensors and devices or through direct signaling of the users. An example is a crowdsourcing service where users can report architectural limits for people with disabilities [21]. In this case, insiders can feed the service with fabricated data to alter the normal behavior of services, e.g., by directing users through specific pathways.

Countermeasures. For the sake of completeness and clarity, let us start from the literature regarding "classic" threat scenarios. Cho et al. [22] examined how insider attacks can exploit security holes in a trusted network of sensor nodes. This work is of interest for our platform because it shows how even trust-based approaches, in architectures that have to unify many nodes, are not guaranteed to prevent attacks.

In [23], the authors described how access control policies for a database management system can be exploited by insiders when the control restrictions to be enforced may come from different authorities. Shatnawi et al. [24] made a similar analysis but based on the detection of malicious usage of a data source, which is equivalent to our case of a malicious influence of data source services exposed by the SMAll platform.

An interesting work that can be applied to our architecture is [25]. Here the authors implemented a pool of honeypots to catch insiders. A honeypot is an information system resource whose value lies in unauthorized or illicit use of that resource. The high flexibility of honeypots able to play a huge variety of SMAll-compliant services is essential to make insiders expose themselves. Another useful method that can be easily built within SMAll is a reporting system for crowdsensing and crowdsourced data, implemented in [26]. The reporting system is based on the mapping of what the authors called Point of Interest (POI). Each POI and its related data can be added to the system by means of one or more reports. Reports are classified in three different source classes, accordingly to the reputation of the user that collects the data.

Service Behavior and Data Manipulation. As expected, insider threats posed by Managers constitute a more complex scenario. This type of insiders can access and modify the raw data of services as well as manipulating their logic to present altered results. Notably, since in our context the physical world mixes with that of software services, we extend the role of Managers not only to the developers that can modify the actual code of the service but also to conductors and other operators: agents that can access and manipulate the physical devices that feed the services.

The manipulation of these services can have many purposes from the point of view of an insider. For example, during the development of SMAll we interacted with many industrial partners, among which there were some public transportation companies that provided real-time positioning of their vehicles. However, some of these companies did not report the actual position of buses and instead published fake positions to mirror the exact planned schedule. In another case the service worked intermittently. In the first case, the company provided fake data to protect itself against possible penalties due to delays, in the second case the positioning service went down for certain rides due to drivers that disabled the in-vehicle positioning devices either for fraudulent purposes (to avoid being scrutinized) or even for shallow reasons such as to disable annoying automatic voice announcements.

Countermeasures. Interesting works tackle the issue of how to predict insiders activities. Ho et. al. [27] implemented a detection mechanism for single users based on analyzes of changes on the writing style of the user after an attack occurred, using machine learning algorithms. Althebyan [28] implemented a prediction model based on graph theory approaches, to push alert once a detection risk mechanism finds that users are performing actions that might lead to compromise the system services.

Studies also exist aimed at discovering malicious command execution. Among the most relevant works, Kamra et al. [29] and Mathew et al. [30] focus on the analysis of anomalous commands executed on databases. In particular, they proposed a syntax analysis system to detect anomalous queries; the former analyzed the submitted SQL queries, while the latter focused on data retrieved from queries. Doss and Tejay [31] conducted a similar investigation as a field study within an enterprise, where analysts were monitored while performing their jobs. Again, these results can be readily applied in our architecture, especially considering that tier I services will in any case be monitored by probes needed to build Business Intelligence services of the second tier.

In principle, the SMAll service deployment interface can verify the correctness of an application before accepting it. In practice, this operation is very hard to perform. One indicator of correctness is the compliance to a template of acceptable interfaces for the kind of service the application provides. However, it is very difficult to define templates strict enough to allow sensible compliance checks, but general enough to avoid hindering the deployment of legitimate services.

Another important detection strategy that we considered is to implement a mechanism that could guarantee, in every moment, a reproducibility of the results of a service. With provenance certifications of raw data and their propagation to results, it is possible to implement a reference monitor to verify compliance between results and expected values. In case of conflicts between the declared results and the actual ones, SMAll could discover what has been tampered with: the source data, or the service logic. This detection can also feed a data trustworthiness rating system.

Finally, another way to check correctness is to look at the actual behavior of the application, as it is common in anti-malware checks. These techniques are far from infallible, and their scope falls much shorter than what is required in our setting. Indeed, in this context a malicious behavior can be a subtle deviation from the correct calculation [32], which is far more difficult than the detection of traditional malicious behaviors (e.g., damaging or selfreplicating ones). Promising techniques, which can benefit from the execution of all the services on the SMAll 
platform, are those based on the aggregation of multidomain information [33], [34].

\section{Travel}

Services in the Travel category orchestrate Information ones to provide highly coordinated functionalities to users. Since the services in this category heavily rely on composition to provide their functionalities, their main concerns regard their workflow.

Service Workflow Manipulation. Managers can modify the expected flow of information among services for many purposes. As an example, consider the Manager of a service called Bus ETA that predicts bus arrivals. In its calculations, Bus ETA uses three source-services, respectively for traffic, GPS positioning, and weather forecasts. Although the Manager preserves the logic (i.e., the behavior) of the Bus ETA service, by simply changing the workflow, i.e., the bindings of the Bus ETA to the other services, she can make (some) of the sources unreachable, either completely disabling the Bus ETA service or modifying the resulting output due to missing data.

Countermeasures. SMAll already provide tools to contrast service workflow manipulations through the helper services Dispatcher and Business Policies (Fig 1). Indeed, when Managers deploy their services in SMAll, they also define the related access rules (stored and retrieved in the Business Policies service). Then, all workflow compositions pass through the Dispatcher service that logs them and enforces the established access policies. In this way, unexpected workflows are detected, logged, and (depending on the access rules) forbidden. The monitoring capabilities of the Dispatcher can also be enhanced with techniques like [35], where the authors propose an analysis to detect malicious workflows and [36], that employs machine learning engines similar to the ones used in dynamic malware analysis to detect malicious workflows. Finally, based on service specifications, we can create workflow graphs for strategic mitigation [37].

Another promising approach comes from the field of Choreographic Programming [38]. The use of choreographies to implement workflows among services is relatively new [39]. We deem choreographies an effective prevention tool that lets partners agree on a formal definition of their workflows, which can be later compiled into their respective, compliant services. Moreover, in the dynamic context of SMAll, tools like [40] can aid partners in updating their agreed workflows even at runtime (i.e., without stopping their running services). These updates would be still conditioned to a general agreement and maintain the same guarantees of the original services.

Mitigation techniques can be also developed following e.g., [41]. The idea would be to develop a SMAll helper service that monitors workflows and, once an attack by an insider is discovered, it appropriately redirects the workflow to avoid further damage.
Composition of Unverified Services and Data. In the context of mobility, verified information is of paramount importance. However, in a service-oriented architecture, the tricky part to deal with is that a service invocation can be seen as a collection of workflows. These workflows can compose many levels of services, each processing and modifying the data before its final destination. These services inherently include the logic of the composed services and, by extension, also the possible manipulations executed by insiders. As an example, consider a journey planner that uses a real-time traffic report service to avoid traffic jams and roadblocks. Since the journey planner directly integrates the information from the traffic report service, manipulating information of the latter alters the solutions of the journey planner, diverting travelers towards certain pathways. This case presents an interesting nuance: the insider is not a direct Manager of the considered service (i.e., the journey planner), instead it is the Manager of a composed service (the traffic report) that twists its contribution to alter the behavior of the planner. In this context also trustability, provenance, and trustworthiness of data and/or services should be considered as possible targets of attacks. For example, tampering with data provenance is a source of attack [42] that in a MaaS scenario can see malicious operators claiming to publish genuine data of a competitor, actually forging them.

Interfering with the certification of data trustworthiness is another possible vector. In this case, it is very difficult to block attacks in which, e.g., the creator advertises a data source of given quality, but then exposes a degraded version to keep the advantage of more precise/timely information for herself. A related trustworthiness scenario is that of an insider who succeeds in registering a rogue service. For example, a modified travel planner could deflect routes to favor or damage certain businesses; a modified delay-checking application could hide or amplify violations of agreed service levels.

Countermeasures. A service must support the provision of different sources of data along with their associated metadata (e.g., used to verify their provenance). However, SMAll shall also provide techniques, embodied by helper services, to transform those data into verified information. Different approaches can be taken to support a solution for the problem of recognizing the source of a data stream. Literature agrees [43] that the requirements for a provenance management system are: Verifiability: a provenance system should be able to verify a process in terms of the actors (or services) involved, their actions, and their relationship with one another; Accountability: an actor (or service) should be accountable for its actions in a process. Thus, a provenance system should record in a non-repudiable manner any provenance generated by a service; Reproducibility: a provenance system should be able to repeat a process and possibly reproduce a process from the provenance stored; Preservation: a provenance system should have the ability to maintain provenance information for an extended period of time. This is essential for applications run in an enterprise system; Scalability: 
given the large amounts of data that an enterprise system handles, a provenance system needs to be scalable; Generality: a provenance system should be able to record provenance from a variety of applications; Customizability: a provenance system should allow users to customize it by setting metadata such as time, events of recording, and the granularity of provenance.

In these regards, it would be useful to deploy technologies to certify the metadata related to a data stream and manage its validity during time and re-elaboration [44]. According to works like [45], this problem could be solved only with the creation of a public-private key system for data stream certification. A good reference is the system developed in [46], describing a cryptographic provenance verification approach for ensuring data properties and integrity for single hosts. Specifically, the authors designed and implemented an efficient cryptographic protocol that enforces keystroke integrity. This kind of protocols can be integrated as a helper service in SMAll. However, publickey schemes are known for their significant computational load, thus existing techniques may not be suitable for highrate, high-volume data sources. Moreover, there could be the need for an algorithm for the provenance of composed data. In some cases, data originated from the composition of raw (or otherwise lower ranked) sources should be accompanied by suitable metadata for verifying the provenance of the input values, in a cryptographically strong way. In the context of SMAll, it could be important and useful to capture and understand the propagation of data.

The combination of metadata- with key-propagation management can guarantee a good level of trust in provenance management systems. Works in the direction of [47] discuss how to support provenance awareness in spatial data infrastructure and investigates key issues including provenance modeling, capturing, and sharing, useful to implement key propagation systems.

Finally, we address trustability, provenance, and trustworthiness of services and/or data.

Trustability needs to be measured by indicators for data quality and service behavior. Values for these indicators come from a variety of considerations on basic data sources. However, it is challenging to define algorithms for source evaluation based on data resulting from services aggregating and orchestrating other sources [48], [49]. Ascertaining provenance means ensuring that the source of data is verifiable, i.e., that it corresponds to the one declared in the process of creation. Trustworthiness is intended as the possibility to ascertain the correctness of the information provided by a data source, which is loosely related to provenance [50]. Ideally, but infrequently, data samples can be independently measured by different users, thus allowing cross-checking and error correction. For original data, i.e., provided by its creator, the trustworthiness score is usually derived from the reputation of the creator. Clearly, guaranteeing data quality, provenance and trustworthiness is not enough, it is necessary to ensure that the computation is correct and that no useful results are hidden (completeness).

\section{User}

The last category of services of tier I is not specific to mobility but it contains essential functionalities for the other two categories. The most representative case is that of User Profiling and Management. User profiling is not required to create services for mobility, but it has become essential to ensure usability, to provide user assistance, and to even anticipate and plan for the next movements of the user (cf. Google Now ${ }^{3}$ ).

Data theft. Here, the most obvious threat regards the possibility of stealing information derived from the profile dataset, such as preferences, recordings of movements, orders and payments.

Countermeasures. In our setting, a possible approach is to empower the user with control over its profile and the related access policies [51].

\section{MaaS Stack | Tier II}

\section{Business Intelligence}

The second tier of the MaaS Stack adds a new category next to the ones of the first tier: Business Intelligence, i.e., services exclusively dedicated to provide insight on the usage and performances of services of the first tier.

This services can implement any kind of data mining algorithm useful for monitoring the profitability, sustainability, and reliability of the provided services, as well as for determining trends and making predictions on future usage, for capacity planning and policy definition.

Business Intelligence Data Theft. Business Intelligence analyzes are important source of sensitive information for insiders (also in this case Managers with privileged access) that could expose relevant data to third parties. Indeed, without Business Intelligence services it would be very difficult or even impossible for insiders to obtain such data, that otherwise would require the access to massive amounts of private information over long periods.

Managers of Business Intelligence services can apply targeted analyzes to infer reserved information, such as policies and business strategies of their company. An example of this type of attack is what we simulated in [52], where by just analyzing the database of validated tickets of a public transport company of the urban area of Bologna, we were able to reconstruct the distribution of the various types of tickets in the different zones of the city.

Countermeasures. SMAll serves the purpose of mediating the access to relevant data for Business Intelligence. Every operator wishing to obtain statistics or performance indicators about its own services can freely create instances of the platform-approved analytics services.

Regarding mitigation, the most effective way to hinder the possibility to misuse Business Intelligence services is to properly sanitize the datasets and to control the workflow of this information. These techniques [53] aim

3. https://www.google.com/search/about/learn-more/now/ 
to prevent insiders from correlating Business Intelligence services with external data sources to derive hidden patterns or de-anonymize sensitive information.

\section{MaaS Stack | Tier III}

The third tier of the MaaS Stack is that of MaaS operators, i.e., eMobility operators that use services of other companies, traded within a federated market. In our case, SMAll gives support to such a market but the creation of dynamic federations of MaaS operators rises specific threats within SMAIl (and MaaS markets in general).

In this scenario the main issues to consider are:

- Data service management to avoid manipulation, impersonation, and sensitive pattern discovery (Prevention and Detection);

- Service workflow management to monitor invocation trends of services (Mitigation and Detection);

- Service quality and trustability management to verify the correctness of the service results (Prevention and Detection)

Indeed, the PaaS layer in SMAll differs from most PaaS solutions. Traditionally PaaS provides offer execution environments that isolate tenants. On the contrary, SMAll is built to ease the publication, integration, and orchestration of services owned by different operators.

A simple example to clarify this characteristic is a onestop ticketing application that orchestrates:

- a dynamic planner service providing routing options;

- a user profile manager to sort them according to user preferences;

- a real-time availability seat reservation service;

- a set of services for payment.

The hierarchy of the ticketing service spans many layers, e.g., it integrates the dynamic planner that, in turns, orchestrates many services for static (mapping, timetables) and real-time data (delays, planned extraordinary events, disruptions). The composition of services forms a tree of dependencies that reaches the level of raw-data information services, possibly branching within the domains of different companies.

Since SMAll aims at supporting this kind of interoperability, we argue that it shall also assume responsibility for the trustworthiness and reliability of the services; this is unusual for traditional PaaS [54]. Moreover, access control policies can be heterogeneous, exchanged data can have different sensitivity levels, and the agents can be competing operators.

Clearly, the main insider threat for this scenario comes from the service providers themselves, the MaaS operators. The malicious goals can be of various kinds, spanning from the de-legitimization of services of competing operators, to the theft of stored information such as policies or business strategies, to insiders that apply mining techniques to infer these information using the data available from their vantage point.

We now proceed by focusing our analysis on the relevant insider threats within the categories of the third tier of the MaaS Stack.

\section{Roaming and Clearing}

SMAll aims at providing interoperability between different operators. In this context, interoperability means that it is possible to implement ticketing systems which seamlessly work on different operators across their zones of influence. As mentioned in $\S 2$, this concept (and the category of services that supports it) takes the name of Roaming. Usually, to support at a business level the roaming for users among operators, business agreements should be put into place to implement a Clearing system for the redistribution of profits between transport operators. In this Section, we consider threats as directed to the Clearing category since it comprises also the threats to the Roaming one.

Pattern Extraction. As analyzed in [52], the need for Clearing services is satisfied through a centralized (federation-wise) system able to collect all the different data sources from different operators and to perform economic evaluations. A centralized clearing system scenario is typically based on a central database that collects all the ticket validation data from every public transport operator. This database is used both to perform economic evaluations to redistribute profits and to store a permanent proof of the validity of this evaluation. The clearing system must fulfill an effective trade-off between public verifiability of the correctness of its operation and protection of sensitive data provided by operators. As the last cited work shows, an insider can perform data mining analysis and pattern discovery on the tickets datasets in order to retrieve sensitive information about business strategies and perform unfair competition.

Countermeasures. To counteract Pattern Extraction, it is possible to deploy sanitization techniques [55] able to mask the data enough to deny the possibility to perform pattern analysis. These sanitization techniques balance masking sensitive data and keeping enough properties and information required to perform the economic evaluations. In order to do what we described, we could assemble an anonymization system, that combines masking techniques for the raw dataset (once deployed in the centralized database clearing system) and a differential privacy engine able to introduce a certain amount of noise and prevent exploit techniques as cross-combining data with external ones.

\section{Access Control and Service Level Agreement}

Service Level Agreement (SLA) and Access Control (AC) services in SMAll are meant to throttle the invocation of tier I services provided by an operator on the basis of commercial agreements with other operators. It is possible to see SLA as a contract ruling the quantity or rate of invocation of each service, and AC as a contract ruling the quality or the set of provided data or services. Obviously, malicious insiders may try to circumvent these limitations.

Countermeasures. When a SLA or an AC policy is in place, all service invocations must be tracked (or even proxied) by an infrastructural service provided by SMAll. 
This makes evading enforcement difficult. The most common vulnerability in this context is not tied to policy enforcement, however, but rather to policy specification. To this end, SMAll could restrict acceptable policies to those drafted with an internal helper service, following a standard framework, and formally verifying their soundness before applying them. Access control models and formal policy specification languages have been around for some time [56], [57], and they have evolved into sophisticated, standardized models like ABAC [58], [59]. Inadequate (but consistent) policy definitions due to poor understanding of the federation interactions or to carelessness cannot be tackled at this level; logging and auditing facilities integrated in SMAll provide valuable feedback at run-time about the effectiveness of installed policies.

\section{Business Intelligence}

Similarly to tier II, in tier III we have a category of services dedicated to business intelligence. The difference with respect to the services of the second tier is that here the analyzes span data belonging to a multitude of operators. Indeed, as it happens for clearing services, the business intelligence services of the third tier relate to the management of data, statistics, and administration of services shared among operators. The availability of such aggregated data can give free access to companies (seen as federated insiders) to data and analyzes of competitors. Referring again the case of the dynamic route planner as a running example, the service can use real-time data of different companies to take into account the average delays of transport vehicles in the calculation of its solutions. The averaged delays are the result of a business intelligence service that collects all the delays of a route within a specific area that involves several operators and calculates the delays. Finally, the recorded delays are collected into a shared dataset accessible by all the participants.

In this example, an insider can use the collected dataset to find out where the competitors operate with bigger delays and profit from this information by exposing their faults to the regional administration. Insiders can also expose cartels where operators systematically provide a bad service during rush hours to favor a specific company (e.g., because they hold some economic interest in it). Finally, the insiders can also find out if an operator hides delays making analysis on the correspondent road conditions (e.g., showing that buses could not sustain certain speeds since their routes were jammed).

Countermeasures. All the countermeasures for this kind of attacks are based on a trade-off between the amount of sensitive data preserved and utility of the queries. Different anonymization and sanitization techniques have been proposed for complex datasets, but since in SMAll Business Intelligence services share the results of queries, we need to introduce a measure that indicates the maximum amount of anonymized information such that the queries still work.

Different works proposed metrics for the evaluation of the amount of privacy preserved in specific dataset. A measure introduced in [60] defined an evaluation metric about the presence of pattern in a dataset called $\delta$-presence. We can use this metric to evaluate the presence of a specific patterns in the shared dataset. Another interesting work in this direction is [61] which operates by complementing existing techniques with post randomization methods.

\section{Conclusions}

In this paper, we presented the concept of Mobility as a Service and how MaaS operators shall facilitate the dynamic provisioning of multi-modal transportation to their users. To support such flexibility we are developing a federated marketplace of services called SMAll, aimed at harmonizing data flows and service invocations.

This kind of federated platform is particularly sensitive to insider threats, which emerge at different layers, targeting both the constituent components provided by users and operators and the services provided by the platform itself.

The MaaS Stack, our tiered view on the components of MaaS markets, allowed us to treat in isolation the security issues of each tier. Often, these issues turn out to be instances of well-known threats in the fields of cloud computing, service-oriented architectures, supply chain management, and trusted business partnerships.

In principle, the platform allows to implement contextspecific versions of the solutions proposed in the literature regarding the aforementioned fields, as well as novel solutions inspired by their cross-fertilization. We argue that the central role of SMAll in mediating every interaction and in collecting their traces makes the platform fit to host solutions to the presented security issues of MaaS markets.

The effectiveness of the proposed approaches will be experimentally validated in the near future, following the completion of the platform in all its parts, and the deployment of real-world services on it.

\section{REFERENCES}

[1] R. Buyya, C. S. Yeo, S. Venugopal, J. Broberg, and I. Brandic, "Cloud computing and emerging IT platforms: Vision, hype, and reality for delivering computing as the 5th utility," $F G S$, vol. 25, no. 6, pp. $599-616,2009$.

[2] S. Pippuri, S. Hietanen, and K. Pyyhtiä, "Maas finland." http: //maas.fi/.

[3] B. Rochwerger, D. Breitgand, E. Levy, A. Galis, K. Nagin, I. M. Llorente, R. Montero, Y. Wolfsthal, E. Elmroth, J. Caceres, et al., "The reservoir model and architecture for open federated cloud computing," IBM JRD, vol. 53, no. 4, pp. 4-1, 2009.

[4] R. Buyya, R. Ranjan, and R. N. Calheiros, "Intercloud: Utilityoriented federation of cloud computing environments for scaling of application services," in AAPP, pp. 13-31, Springer, 2010.

[5] M. Kandias, N. Virvilis, and D. Gritzalis, The Insider Threat in Cloud Computing, pp. 93-103. Springer, 2013.

[6] P. Zhou, Y. Zheng, and M. Li, "How long to wait?: predicting bus arrival time with mobile phone based participatory sensing," in Proceedings of MobiSys, pp. 379-392, ACM, 2012.

[7] S. Mirri, A. Melis, C. Prandi, and M. Prandini, "Crowdsensing for smart mobility through a service-oriented architecture," in ISCC, p. 5, IEEE, 2016.

[8] S. Giallorenzo, A. Melis, and M. Prandini, "Smart Mobility for All," tech. rep., University of Bologna, 2016.

[9] M. Mouly, M.-B. Pautet, and T. Foreword By-Haug, The GSM system for mobile communications. Telecom publishing, 1992. 
[10] H. Mun, K. Han, C. Yeun, and K. Kim, "Yet another intrusion detection system against insider attacks," Proc. of SCIS, 2008.

[11] V. Stavrou, M. Kandias, G. Karoulas, and D. Gritzalis, Business Process Modeling for Insider Threat Monitoring and Handling, pp. 119-131. Cham: Springer International Publishing, 2014.

[12] L. Flynn, G. Porter, and C. DiFatta, "Cloud service provider methods for managing insider threats: Analysis phase ii, expanded analysis and recommendations," 2014.

[13] W. R. Claycomb and A. Nicoll, "Insider threats to cloud computing: Directions for new research challenges," in ACSAC, pp. 387-394, IEEE, 2012.

[14] B. Schneier, Secrets and lies: digital security in a networked world. John Wiley \& Sons, 2011.

[15] N. Nostro, A. Ceccarelli, A. Bondavalli, and F. Brancati, "Insider threat assessment: A model-based methodology," $A C M$ SIGOPS, vol. 48, no. 2, pp. 3-12, 2014.

[16] T. Casey, "A field guide to insider threat," tech. rep., Intel, 2015.

[17] G. Silowash, D. Cappelli, A. Moore, R. Trzeciak, T. J. Shimeall, and L. Flynn, "Common sense guide to mitigating insider threats 4th edition," tech. rep., DTIC Document, 2012.

[18] M. Bishop, "Position: Insider is relative," in Proceedings of Workshop on New security paradigms, pp. 77-78, ACM, 2005.

[19] J. Hunker and C. W. Probst, "Insiders and insider threats-an overview of definitions and mitigation techniques.," JoWUA, vol. 2, no. 1, pp. 4-27, 2011

[20] A. Shabtai, Y. Elovici, and L. Rokach, A survey of data leakage detection and prevention solutions. Springer, 2012.

[21] S. Mirri, A. Melis, C. Prandi, and M. Prandini, "A microservice architecture use case for persons with disabilities," in CVSJ, p. 5, Hindawi, 2016.

[22] Y. Cho, G. Qu, and Y. Wu, "Insider threats against trust mechanism with watchdog and defending approaches in wireless sensor networks," in SPW, pp. 134-141, IEEE, 2012.

[23] M. Gertz and S. Jajodia, Handbook of database security: applications and trends. Springer, 2007.

[24] N. Shatnawi, Q. Althebyan, and W. Mardini, "Detection of insiders misuse in database systems," in ICECS, vol. 1, 2011.

[25] L. Spitzner, "Honeypots: Catching the insider threat," in CSAC, pp. 170-179, IEEE, 2003.

[26] S. Mirri, A. Melis, C. Prandi, and M. Prandini, "A serviceoriented approach to crowdsensing for accessible smart mobility scenarios," in Proceedings ICCTS, p. 5, IEEE, 2016.

[27] S. M. Ho, J. T. Hancock, C. Booth, M. Burmester, X. Liu, and S. S. Timmarajus, "Demystifying insider threat: Languageaction cues in group dynamics," in HICSS, pp. 2729-2738, IEEE, 2016.

[28] Q. Althebyan, Design and analysis of knowledge-base centric insider threat models. ProQuest, 2008.

[29] A. Kamra, E. Terzi, and E. Bertino, "Detecting anomalous access patterns in relational databases," The VLDB Journal, vol. 17, no. 5, pp. 1063-1077, 2008

[30] S. Mathew, M. Petropoulos, H. Q. Ngo, and S. Upadhyaya, "A data-centric approach to insider attack detection in database systems," in RAID, pp. 382-401, Springer, 2010.

[31] G. Doss and G. Tejay, "Developing insider attack detection model: a grounded approach," in ISI, pp. 107-112, IEEE, 2009.

[32] A. Moser, C. Kruegel, and E. Kirda, "Limits of static analysis for malware detection," in ACSAC, pp. 421-430, Dec 2007.

[33] H. Eldardiry, E. Bart, J. Liu, J. Hanley, B. Price, and O. Brdiczka, "Multi-domain information fusion for insider threat detection," in SPW, pp. 45-51, May 2013.

[34] Q. Althebyan, R. Mohawesh, Q. Yaseen, and Y. Jararweh, "Mitigating insider threats in a cloud using a knowledgebase approach while maintaining data availability," in ICITST, pp. 226231, IEEE, 2015

[35] B. G. Schlicher, L. P. MacIntyre, and R. K. Abercrombie, "Towards reducing the data exfiltration surface for the insider threat," in HICSS, pp. 2749-2758, IEEE, 2016.

[36] M. D. Ernst, "Static and dynamic analysis: Synergy and duality," in WODA, pp. 24-27, Citeseer, 2003.

[37] V. B. Velpula and D. Gudipudi, "Behavior-anomaly-based system for detecting insider attacks and data mining," IJRTE vol. 1, no. 2, pp. 261-266, 2009.
[38] F. Montesi, Choreographic Programming. PhD thesis, IT University of Copenhagen, 2013.

[39] S. Giallorenzo, Real-World Choreographies. PhD thesis, Università degli studi di Bologna, 2016.

[40] M. Dalla Preda, S. Giallorenzo, I. Lanese, J. Mauro, and M. Gabbrielli, "AIOCJ: A choreographic framework for safe adaptive distributed applications," in SLE, pp. 161-170, Springer, 2014

[41] H. G. Goldberg, W. T. Young, A. Memory, and T. E. Senator, "Explaining and aggregating anomalies to detect insider threats," in HICSS, pp. 2739-2748, IEEE, 2016.

[42] Y. L. Simmhan, B. Plale, and D. Gannon, "A survey of data provenance in e-science," SIGMOD Rec., vol. 34, pp. 31-36, Sept. 2005.

[43] P. Groth, M. Luck, and L. Moreau, "A protocol for recording provenance in service-oriented grids," in PDS, pp. 124-139, Springer, 2004.

[44] W.-T. Tsai, X. Wei, Y. Chen, R. Paul, J.-Y. Chung, and D. Zhang, "Data provenance in SOA: security, reliability, and integrity," SOCA, vol. 1, no. 4, pp. 223-247, 2007.

[45] Y. L. Simmhan, B. Plale, and D. Gannon, "A survey of data provenance techniques," CSD, IU, Indiana, vol. 47405, 2005

[46] K. Xu, H. Xiong, C. Wu, D. Stefan, and D. Yao, "Dataprovenance verification for secure hosts," DSC, vol. 9, pp. 173183, March 2012

[47] L. He, P. Yue, L. Di, M. Zhang, and L. Hu, "Adding geospatial data provenance into SDI - a service-oriented approach," AEORS, vol. 8, no. 2, pp. 926-936, 2015.

[48] C. Falge, B. Otto, and H. Österle, "Data quality requirements of collaborative business processes," in HICSS, pp. 4316-4325, Jan 2012.

[49] S. Dustdar, R. Pichler, V. Savenkov, and H.-L. Truong, "Qualityaware service-oriented data integration: Requirements, state of the art and open challenges," SIGMOD Rec., vol. 41, pp. 11-19, Apr. 2012.

[50] C. Dai, D. Lin, E. Bertino, and M. Kantarcioglu, SDM, ch. An Approach to Evaluate Data Trustworthiness Based on Data Provenance, pp. 82-98. Berlin, Heidelberg: Springer, 2008.

[51] K. K. Antti Poikola and H. Honko, "Mydata a nordic model for human-centered personal data management and processing," tech. rep., Ministry of Transport Finland, 2014.

[52] F. Callegati, A. Campi, A. Melis, M. Prandini, and B. Zevenbergen, "Privacy-preserving design of data processing systems in the public transport context," Pacific Asia Journal of the Association for Information Systems, vol. 7, no. 4, 2015.

[53] B. R. Mistry and A. Desai, "Privacy preserving heuristic approach for association rule mining in distributed database," in ICIIECS, pp. 1-7, IEEE, 2015.

[54] S. E. Madnick, R. Y. Wang, Y. W. Lee, and H. Zhu, "Overview and framework for data and information quality research," $J$. Data and Information Quality, vol. 1, pp. 2:1-2:22, June 2009.

[55] D. Molnar, B. Livshits, P. Godefroid, and P. Saxena, "Automatic context-sensitive sanitization," Nov. 25 2014. US Patent $8,898,776$

[56] R. S. Sandhu, E. J. Coynek, H. L. Feinsteink, and C. E. Youmank, "Role-based access control models yz," IEEE computer, vol. 29, no. 2, pp. 38-47, 1996.

[57] N. Damianou, N. Dulay, E. Lupu, and M. Sloman, "The ponder policy specification language," in IWPDSN, POLICY '01, (London, UK, UK), pp. 18-38, Springer-Verlag, 2001.

[58] V. C. Hu, D. Ferraiolo, R. Kuhn, A. R. Friedman, A. J. Lang, M. M. Cogdell, A. Schnitzer, K. Sandlin, R. Miller, K. Scarfone, et al., "Guide to attribute based access control (abac) definition and considerations (draft)," NIST Special Publication, vol. 800, no. 162,2013

[59] R. Sandhu, "Attribute-based access control models and beyond.," in ASIACCS, p. 677, 2015.

[60] M. E. Nergiz, M. Atzori, and C. Clifton, "Hiding the presence of individuals from shared databases," in SIGMOD, pp. 665-676, ACM, 2007.

[61] T. S. Heydt-Benjamin, H.-J. Chae, B. Defend, and K. Fu, "Privacy for public transportation," in IWPET, pp. 1-19, Springer, 2006. 